\title{
Ethnologies
}

\section{Les itinéraires de l'exclusion pour un groupe de sans-abri}

\section{Laurent Jérôme}

Volume 24, numéro 1, 2002

Espace

Space

URI : https://id.erudit.org/iderudit/006532ar

DOI : https://doi.org/10.7202/006532ar

Aller au sommaire du numéro

Éditeur(s)

Association Canadienne d'Ethnologie et de Folklore

ISSN

1481-5974 (imprimé)

1708-0401 (numérique)

Découvrir la revue

Citer cet article

Jérôme, L. (2002). Les itinéraires de l'exclusion pour un groupe de sans-abri. Ethnologies, 24(1), 101-117. https://doi.org/10.7202/006532ar

\section{Résumé de l'article}

Ce texte vise à rendre compte des interactions entre les individus marginalisés d'un groupe de sans-abri de la ville française de Metz (Est de la France) et les lieux urbains qu'ils investissent quotidiennement. Exclus de la société dominante, ces sans-abri vivent leur marginalité en intégrant de nouvelles valeurs et de nouvelles références culturelles qui prennent forme dans une sous-culture étiquetée comme déviante. Cette mise en marge entraîne une identification et un engagement de leur être au monde à d'autres lieux dont la mise en relation forme un itinéraire de l'exclusion, et donc un espace urbain marginal.
Ce document est protégé par la loi sur le droit d'auteur. L'utilisation des services d'Érudit (y compris la reproduction) est assujettie à sa politique d'utilisation que vous pouvez consulter en ligne.

https://apropos.erudit.org/fr/usagers/politique-dutilisation/ 


\title{
LES ITINÉRAIRES DE L'EXCLUSION POUR UN GROUPE DE SANS-
} ABRI

\author{
Laurent Jérôme \\ Groupe d'Études Inuit et Circumpolaires \\ Université Laval
}

\begin{abstract}
« Nous réservons le terme "lieu anthropologique " à cette construction concrète et symbolique de l'espace qui ne saurait à elle seule rendre compte des vicissitudes et des contradictions de la vie sociale mais à laquelle se réfèrent tous ceux à qui elle assigne une place, si humble ou modeste soit elle» (Augé 1992: 68)
\end{abstract}

Ce jour-là, quelques photographies de sans-abri tapissaient les murs de l'espace «exposition" de la bibliothèque universitaire de Metz. Le thème semblait classique, au sens médiatique, et dérangeant, au sens populaire. En définitive, c'était un thème à la mode pour la période ; en France, durant quatre mois, de novembre à mars, les sans logis, sansabri, sans domicile fixe, clochards et autres vagabonds ou errants font l'objet de toutes les attentions, même si personne ne sait vraiment comment les nommer, ni quelles réalités sociales se cachent derrière ces termes vagues et impersonnels. Ils ne bouleversent pas nos habitudes, mais réveillent chaque année la conscience collective, la solidarité nationale.

Les photographies étaient accompagnées d'une légende explicative nécessaire à une compréhension du but recherché par le photographe ; nécessaire aussi pour mettre un prénom ou un surnom sur le visage du sujet photographié ; photos en noir et blanc, de vies, de morts, d'espoir, de misère ou de haine; photos de corps abîmés, prostrés, souillés ou enivrés, derrière lesquels nous pouvions aisément reconnaître les quartiers, les rues ou les bâtiments les plus populaires de notre propre ville. Ces photographies, extraites d'une ouvre de dénonciation sociale (Taesch et Robert 1997), nous ont personnellement touché et nous n'avons eu d'autre choix que de laisser paraître, ce jour-là, notre 
subjectivité. Nous avons alors décidé de l'objectiver en tentant de livrer notre propre vision de ce phénomène de l'exclusion.

Celui-ci ne constitue plus aujourd'hui un thème étranger à l'analyse des phénomènes sociaux contemporains. Il est même possible d'affirmer que ces modes particuliers de rapport au social pourraient être interprétés comme l'envers ou l'échec de l'intégration, de l'assimilation ou de l'insertion sociales touchant aussi bien des individus que des groupes complets. Bien que marginalité et exclusion fassent référence à des univers partiellement différents, ces deux notions renvoient à la fois au processus dynamique de mise à la marge et au sens de celui-ci. Être marginalisé ou exclu suppose une distanciation du social dans le sens d'une détérioration plus ou moins complète des conditions de vie au regard des normes et des valeurs dictées par la société dominante (Bourdieu 1979 ; Grignon et Passeron 1985). Ainsi, la notion de distanciation introduit la dimension socio-spatiale de la marginalité et de l'exclusion : être à la marge renvoie à la fois à un espace périphérique mais aussi à l'insertion de celui-ci dans les rapports sociaux de la société.

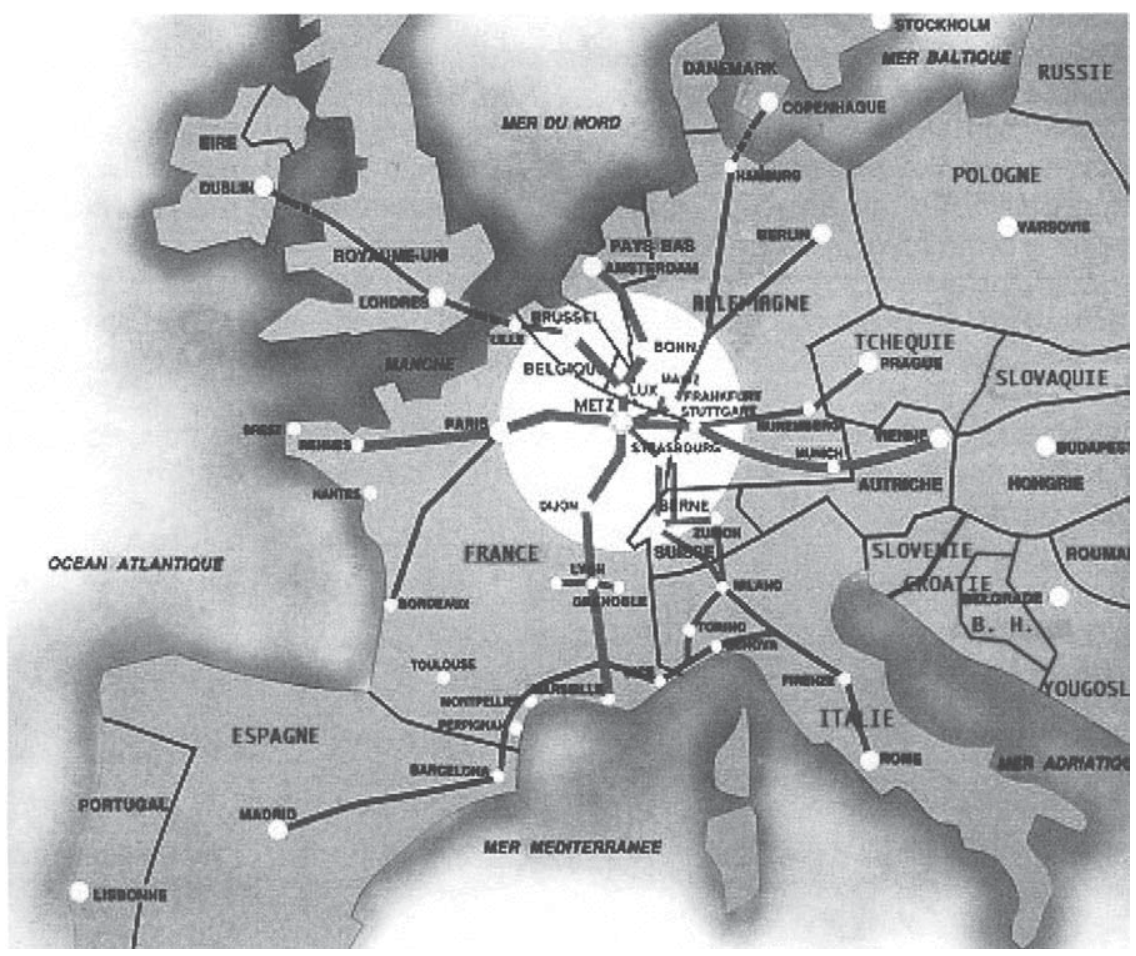

Situation géographique de la ville de Metz dans l'espace européen 
En ce sens, notre propos est d'évoquer un rapport particulier à l'intérieur de cette relation au social déjà singulière. Nous proposons de mettre en lumière quelques aspects d'une forme précise de cette mise à la marge à travers le phénomène de l'itinérance urbaine. La compréhension des dynamiques sociales et culturelles de la population itinérante de nos villes pose des difficultés particulières : flou des définitions, absence d'informations systématiques, difficultés d'identifier et de rencontrer des répondants, stigmate social et crainte des conséquences négatives, problèmes éthiques d'ordres divers (Laberge et Roy 1994). Nous avons expérimenté ces difficultés lors d'une enquête de terrain réalisée d'octobre 1999 à avril 2000 au sein de la ville française de Metz (Est de la France) ${ }^{1}$. Ville transfrontalière, elle peut être considérée comme le cœur géographique de l'Europe ; elle accueille ainsi de nombreuses populations en transit vers l'Allemagne, la Belgique, l'Angleterre ou encore l'Espagne. Elle apparaît souvent comme un point de passage obligé pour de nombreux itinérants; elle est aussi un espace social au sein duquel certains individus marginalisés s'installent et recréent du sens.

\section{Le groupe}

Le groupe que nous avons suivi tout au long de cette enquête vit en intégrant de nouvelles valeurs et références culturelles en marge de la culture dominante. Il s'est formé sur plusieurs années autour de Moïse (52 ans, 19 ans de rue); se sont ensuite successivement greffés Goliath (49 ans, 9 ans de rue), Libellule, seule femme du groupe (43 ans, 8 ans de rue, depuis 6 ans dans le groupe) et David (24 ans, 5 ans de rue, depuis 4 ans dans le groupe $)^{2}$. Les parcours de vie de ces quatre sans domicile fixe ont leurs points communs : «chute » dans la rue après une rupture sociale et/ou professionnelle, nouvelles références et nouvelles valeurs s'inscrivant dans une nouvelle organisation de vie et

1. Une première version de ce texte a été présentée lors du séminaire d'anthropologie de Sylvie Poirier, Les constructions sociales de la nature (Université Laval, 2000-2001). Nous tenons ici à remercier Virginie Vinel, qui a suivi avec implication le déroulement de cette recherche effectuée au sein du département d'ethnologie de l'Université de Metz, Sylvie Poirier et Richard Lioger pour leur disponibilité et leurs lectures attentives et critiques qui ont conduit à l'élaboration de la version finale.

2. Libellule, David, Goliath et Moïse sont des pseudonymes que nos informateurs se sont attribués eux-mêmes lorsque nous avons décidé ensemble de respecter leur anonymat. 
relevant du choix de refuser d'intégrer les réseaux et les structures d'aide sociale. Ces quatre sans-abri pratiquent tous la mendicité dans les endroits les plus fréquentés du centre-ville, ce qui les prive de leurs droits de percevoir des revenus d'assistance sociale. Le groupe, en tant que formation sociale, nous a permis de formuler une lecture d'ensemble des rapports à soi et aux autres, aux institutions, au monde en général. Nous devions comprendre les liens qui unissaient chaque membre, leur histoire à travers leurs parcours de vie ou encore leurs rapports avec la société dominante. D'un point de vue historique, la dynamique de groupe a développé notre compréhension de l'assise des phénomènes culturels liés à la rue ; cet ensemble humain s'est constitué autour d'individus aujourd'hui disparus étiquetés eux aussi comme marginaux. Psychologiquement enfin, le groupe nous a autorisé à mesurer les convergences et les divergences d'avis, la pluralité autant que la similitude des manières de faire, et la place de chacun à l'intérieur de l'unité.

\section{Une ethnographie du néant?}

Qui sont-ils ? Des vagabonds, des SDF ? Patrick Declerck a décidé de les appeler clochards, "parce qu'il faut bien leur donner un nom. Celui-là n'est en rien meilleur que les autres, sinon qu'il renvoie à des images partagées, en France, et par tout le monde. » (Declerck 2001 : 12). Il suffira au lecteur de se reporter à la bibliographie de cet auteur pour se rendre compte, s'il n'en avait pas déjà conscience, de la multitude des études d'ethnologie urbaine (Anderson 1993), de sociologie de la déviance et de l'exclusion (Mercier 1996 ; Clavel 1998 ; Frétigné 1999), de psychologie sociale (Vexliard 1956, 1957) et autres ethnographies menées par des spécialistes en sciences sociales (Gaboriau 1993 ; Garnier-Muller 2000), aussi bien que par ces journalistes qui ont endossé le statut d'ethnographe l'espace de quelques mois, le temps d'une enquête obscure conduite sur le terrain, dans l'ombre de ces corps à bout (voir Prolongeau 1993). La démarche adoptée pour cette recherche est une démarche que l'on peut qualifier de classique. Nous avons choisi d'évoluer avec les membres d'un groupe de sans-abri dans la proximité, de les suivre au quotidien, d'observer en participant. Nous avons entrepris une ethnographie du pauvre conduite sur le terrain, sans bureau ni chaise, avec les lieux publics comme espace de confidences. Nous avons participé, partagé les repas, les attentes, les promenades à travers le quartier, vu de nos propres yeux, observé en situation afin de disposer de données recueillies de première main. Après 
deux mois, nous avions l'impression de faire partie de leur quotidien, d'être le confident, celui à qui, puisqu'il est de l'extérieur, on peut tout dire ou presque. Nous avons partagé leur mode de vie, l'objectif étant de comprendre l'optique de ces marginaux et non de nous transformer en l'un d'eux. À titre d'exemple, nous n'avons jamais passé la nuit à leurs côtés, pensant que nos relations évoluaient suffisamment positivement durant la journée et qu'il était donc inutile de nous intégrer à leur vie nocturne ${ }^{3}$.

Les essais de conceptualisation et de théorisation du «monde de la cloche » tendent globalement à classer et à enfermer le sujet dans une sphère hermétique de comportements à l'aide d'un langage inaccessible, en total décalage avec la clarté apparente de la précarité du vécu quotidien (Moreau de Bellaing et Guillou 1995). Car, s'il faut percevoir ce groupement humain comme une société distincte, développant des références et des stratégies particulières au sein d'un rapport lui-même particulier au social, cette société n'existe que "par défaut, composée d'instables et ponctuels agrégats d'individus plus ou moins isolés dans le silence ou la vocifération de leurs délires éthyliques ». Qu'en est-il alors « du statut épistémologique d'une ethnographie du désordre, du chaos, du néant» (Declerck 2001 : 14), voire de "l'inutilité sociale»(Garnier-Muller 2000)?

\section{Le corps dans l'espace}

Dans un premier temps, notre objectif a été de livrer un regard sur la vie sociale marginale à travers le corps, c'est-à-dire de proposer une lecture sociale des pratiques et des représentations corporelles d'un groupe de sans-abri. Nous avons considéré le corps comme le pivot autour duquel va s'ordonner, en cercles concentriques, toute la vie sociale. Le corps comme axis mundi en quelque sorte. C'est à travers le corporel que se nouent les rapports sociaux les plus fondamentaux. La relation de ces sans-abri avec leur corps nous est en effet apparue comme constante et immédiate. L'importance du corps est telle que l'on peut dire de la vie qu'elle est un corps-à-corps permanent. La relation avec l'extérieur, l'environnement aussi bien que l'expression de son infériorité, passent par le corps, de telle sorte que l'on peut affirmer que la culture est physique (de Meca 1988).

3. La méthode d'enquête de Patrick Gaboriau (1993) et de Hubert Prolongeau (1993) diffère de la nôtre en ce sens qu'ils ont chacun choisi de partager la vie de sans domicile fixe jour et nuit, jusque sur les bancs publics et sous les ponts. 
Il s'agit ici de s'intéresser, non pas aux représentations et aux pratiques corporelles, mais aux positions et aux mouvements de ces corps au sein de l'espace marginal. L'intérêt que nous portons à la relation et à l'investissement quotidiens de l'individu dans certains lieux trouve toute sa pertinence dans ce sens où cette interaction commence avec le corps tout autant qu'elle passe par celui-ci, « une personne incarnée, en chair et en os, munie de sens» (Radice 2000: 15). Cette personne perçoit les choses et porte son attention vers les éléments de son environnement, pour éventuellement le connaître et le maîtriser au cours de ses itinéraires quotidiens (15). Or, c'est bien cette interaction que la théorie de l'engagement (dwelling theory) tend à saisir. Dans ce sens, Ingold suggère " that we [...] follow the lead of Hunter-Gatherers in taking the Human condition to be that of a being immersed from the start, like other creatures, in an active, practical and perceptual engagement with constituents of the dwelt-in world» (Ingold 1996 dans Radice 2000 : 120). La théorie de l'engagement fournit donc un modèle pertinent pour saisir la relation entre les acteurs et les lieux, modèle selon lequel les lieux sont intrinsèquement chargés de sens. Dans le débat qui nous occupe, soit la compréhension des dynamiques spatiales marginales, l'interaction s'inscrit d'abord au sein d'espaces sociaux qui se situent entre le domaine du privé et celui du public.

\section{Corps privés, espaces publics}

Pour de Certeau, il y a espace dès qu'on prend en compte des vecteurs de direction, des quantités de vitesse et la variable du temps. «L'espace est un croisement de mobiles » (1980 : 208). Pour lui, l'espace est un lieu pratiqué ; il serait au lieu ce que devient le mot quand il est parlé. En l'occurrence, au sein de l'espace des membres du groupe, le lieu pratiqué est un lieu public. Nous avons vécu avec nos informateurs sous l'œil des citadins. Nous avons souvent pu constater la curiosité des habitants intrigués et dérangés par les cris ou une musique trop forte. Exposée aux regards, l'existence des gens de la rue est marquée par l'importance du public et de la propriété collective. Ils ont pourtant un sens aigu de la propriété privée et de leur territoire. Le privé est une valeur bien présente dans cette "culture du lieu public ».

En abordant le problème de la vie privée chez les ouvriers du Nord, Olivier Schwartz (1990) se demande si une classe sociale peut réellement être connue si l'on ignore les pôles privés autour desquels se constitue 
une part essentielle de l'existence de ses membres. Il relève deux niveaux de compréhension concernant l'acceptation du terme "privé »:

- D'abord l'idée du secret, de l'intime, du protégé et du caché. Ces notions s'opposent au public en tant que sphère du visible et du regard des autres. Le public désigne ici l'espace de la visibilité sociale et les problèmes qu'il pose aux acteurs. Inversement le privé renvoie au secret.

- Dans notre société, l'opposition privé/public peut aussi jouer dans un autre registre. Le public désignera le commun, ce dont l'usage est ouvert à tous ou qui relève d'une appropriation collective. Inversement, le privé renverra à l'idée de propriété, de possession, de propre.

Le groupe ne peut pas être saisi dans sa totalité si l'on n'a pas constaté chez ses membres cette organisation d'un monde à eux, pensé autour de lieux collectifs, d'objets, de marques qui jalonnent la ville et leurs lieux de passages et de "résidence ». Sa caractéristique principale est justement de s'approprier ce qui est normalement commun et qui d'ailleurs continuera à le rester parallèlement. Ainsi, l'emploi d'adjectifs possessifs dénote l'appropriation du public : «mon garage », « ma gare », « mon entrée d'immeuble », « ma rue », « mon parking »... Cette appropriation peut être individuelle ou collective à l'ensemble du groupe. Les vêtements, les sacs appartiennent à une personne. Certains emplacements sont appropriés par le groupe, comme par exemple le parking de la gare. Ils s'y retrouvent chaque soir avant de rejoindre leur abri de nuit. Celui-ci se caractérise par une appropriation des jardins et squares publics du quartier de la gare. Lorsqu'ils ne dorment pas à ces endroits - la cause étant une alcoolisation excessive et marcher relève alors de l'exploit - ils s'installent devant les entrées d'immeubles ou dans des renfoncements protégés du froid. Mais cette appropriation d'un territoire dissimule en fait une appropriation à leur territoire et une expropriation de tous les autres. Propriétaires forcés de la seule richesse qui leur soit accessible, ils mènent, certes, une vie privée, mais privée de beaucoup de choses.

Malgré une vie en public, ils soulignent l'importance de leur vie privée. Celle-ci s'entend dans un sens restreint, celui d'un domaine réservé à soi et, dans un sens large, de particularité, d'appropriation ou de valeurs du groupe. Il est nécessaire de distinguer ici la notion de «privé » de celle «d'intimité ». Le «privé» ne s'entend pas dans le 
sens de «secret » et «d'intimité » puisque l'organisation de vie se construit dans la sphère $d u$ visible et $d u$ regard des autres.

\section{Les lieux de l'exclusion}

La population de la rue forme une population hétérogène dont l'éclatement apparent révèle un système subjectif et hiérarchisé des individus. Les espaces des marginalités sont humainement et géographiquement circonscrits. Nous avons eu l'occasion de rencontrer des sans-abri dans les gares, sous les arrêts de bus, devant les petits commerces du centre-ville, mais aussi devant les grands centres commerciaux de la périphérie; par ailleurs, d'autres marginalisés se sont installés dans des espaces ouverts comme les grands boulevards extérieurs ou les jardins publics. Dans ces espaces, la prostitution, le travestissement et la toxicomanie cohabitent dans le commerce sexuel à travers un jeu ordonné d'occupation des espaces entre les hommes et les femmes, entre les corps du transsexuel et celui du transformiste.

Les endroits investis par les membres du groupe portent le qualificatif de «public »; ainsi, la voie, le jardin, la place, le banc, les toilettes, l'hôpital, etc... toute une existence quadrillée par un mot. La rue constitue donc le domaine de leur vie privée, même si, à proprement parler, ils ne vivent pas à la rue. Chacun d'entre eux occupe une place déterminée aux alentours du quartier de la gare, dans des endroits délaissés par les citadins, par les commerçants ou par l'administration. La nuit, ils se terrent dans leur renfoncement respectif, à l'abri du vent mais pas du froid, avec une couverture, parfois deux, et leur sac en guise d'oreiller. Le jour, ils empruntent chacun leur itinéraire respectif, marchant irrémédiablement sur le même trottoir, passant dans la même rue chaque jour. Posséder et s'approprier sont deux choses. Ils ne possèdent pas mais s'approprient.

Lorsqu'ils arrivent au centre ville pour commencer leur «travail $»^{4}$, ils s'assoient à la même place, devant la même encoignure de magasin, dans la même position que la veille. Et lorsque nous tentions d'en comprendre les raisons, les réponses étaient toujours identiques : "C’est là que j'suis l'mieux » (Goliath) ou «Y'a qu'eux qui m'disent rien » (Libellule).

4. Il faut sous-entendre ici la pratique de la mendicité, le terme travail étant souvent utilisé par les acteurs pour qualifier cette activité. 
Leur position sur le trottoir nous est apparue comme une préoccupation essentielle. Celui-ci, dans une rue lente pour piétons, apparaît comme l'espace intermédiaire entre l'intérieur des logements et la vie rapide et mécanisée qui ne les préoccupe pas. Ils se déplacent, mangent, boivent, rient et mendient sur le trottoir.

Pendant plusieurs semaines, nous nous sommes demandé comment le marginal évoquait son «chez soi ». Nous parlons de maison, de logement, d'appartement, de studio, mais comment nommer leur espace privé ? Il existe un terme qui permet de l'aborder de l'intérieur. A l'instar de Patrick Gaboriau (1993), nous avons pu constater l'utilisation abondante du terme « coin ». Goliath, Libellule, David ou Moïse disent qu'ils « cherchent un bon coin »: «J'ai le meilleur coin » (David); "J'étais tell'ment bourré que j'pouvais même pu trouver mon coin » (Goliath). Ce terme prend deux significations. Au sens large, il représente le quartier, les alentours, compris comme point de référence. «Dans le coin, t'as tout c'que tu veux » (Libellule); «Depuis l'temps que j'suis dans l'coin » (Moïse). Au sens restreint, le « coin » est l'espace où dorment les enquêtés, il désigne le lieu où ils vont se retirer. C'est un endroit en retrait, en recul, mais, à la différence d'un logement, il est ouvert et dégagé. L'hiver 1999/2000 a été, selon leurs propres termes, « doux» (David), voire "chaud» (Moise). Il n'empêche que les membres du groupe ont accumulé les duvets, les couvertures, les vêtements, les tissus; ils ont passé l'hiver à la rue, sans recourir aux abris collectifs 5 . À la différence des «squatters $»^{6}$, ces marginaux se déplacent avec la totalité de leurs biens et investissent ces différents « coins».

La nuit, les membres du groupe occupent un espace proche, celui du quartier de la gare. Le jour, ils se dispersent dans le centre-ville. Ils se trouvent à plusieurs kilomètres les uns des autres. Leur corps est alors en position d'isolement, si l'on considère que l'isolement est perçu dans un rapport avec les individus d'une même situation sociale. Ils sont seuls face aux jugements, aux paroles blessantes ou, au contraire, aux messages d'encouragement de la majorité sociale. La raison avouée de cette séparation diurne est un meilleur rendement au niveau de la mendicité : «Si on est plusieurs, les gens y donnent moins facilement,

5. Les Centres d'hébergement sont nombreux, y compris dans le centre-ville. De plus, ils sont situés à proximité des parcours de chaque membre du groupe.

6. Les «squatters » investissent les caves, les logements, les usines abandonnés et organisent l'espace de façon à recréer un semblant de logement. 
ils ont plus peur » (Moïse). La nuit, ou tout du moins une partie de la nuit, s'associe à la sociabilité et au groupe, le moment où ils se retrouvent ensemble pour partager certains «gains » de la journée, et notamment les dons en nature tels que la nourriture, mais aussi les anecdotes qui ont pu entraîner un sentiment de nouveauté par rapport à la journée précédente. "J'aime bien quand y'a un truc un peu spécial qui arrive, ça met du piquant pour raconter le soir avec les autres. C'est comme ça que tu t'dis que t'as finalement passé une bonne journée » (Libellule).

Le temps est un facteur essentiel dans la sociabilité du groupe. La pluie, au même titre que la nuit, est synonyme de sociabilité. Ils ne se rendent pas au centre-ville et ne mendient pas les jours de pluie. Ils restent ensemble dans un " coin » abrité et échangent leurs points de vue, boivent, chantent, dansent et interpellent les passants qui leur paraissent sympathiques. Lorsque le temps est favorable, ils se quittent le matin de bonne heure (en général à six heures) et se retrouvent le soir vers 22 heures.

Mais cet investissement de l'espace social public est principalement basé sur un engagement et une mise en relation de lieux chargés de sens. Car, comme le souligne Auguste Berque : «Un lieu, ce n'est pas un espace. L'espace, c'est ce qui met en relation des lieux différents, et qui en cela leur est commun» (1997: 193).

\section{Le lieu anthropologique}

Dans cette problématique de l'itinérance urbaine, le concept de lieu apparaît donc comme essentiel. Même si le terme habiter renvoie à notre notion de l'habitant et donc du logement, il est possible d'affirmer que ces quatre sans-abri habitent la ville de Metz. Il n'est pas question de lieu fermé au regard du public mais de lieux qui, par leur manière d'être investis, deviennent des lieux chargés de sens (Berque 1997) auxquels il est possible d'associer au moins trois caractères communs : ils sont à la fois identitaires, relationnels et historiques (Augé 1992). Identitaires, car les lieux qui jalonnent les parcours et itinéraires sont appropriés par l'acteur qui leur donne un sens ; en ce sens, justement, au sein de cette sous-culture, on va parler du Jardin Public de Goliath, de la Place Publique de Moïse, du Magasin de Libellule... Le lieu est évoqué en référence à celui qui lui donne un sens particulier et qui l'investit. "Le plan de la maison, les règles de la résidence, les quartiers du Village, les autels, les places publiques, la découpe du terroir 
correspondent pour chacun à un ensemble de possibilités, de prescriptions et d'interdits dont le contenu est à la fois spatial et social » (Augé 1992: 69). Michel de Certeau voit dans le lieu l'ordre selon lequel les éléments sont distribués dans des rapports de coexistence et la configuration instantanée de position (1980:173). En un même lieu peuvent donc coexister des éléments distincts et singuliers dont on ne «s'interdit de penser ni les relations ni l'identité partagée que leur confère l'occupation du lieu commun» (Augé 1992 : 71).

Les membres du groupe évoluent dans une configuration d'ensemble dont ils partagent avec d'autres l'inscription sur le sol. En ce sens, les lieux peuvent être considérés comme relationnels à la fois au sein du groupe (les réunions nocturnes communes à la gare), mais aussi en marge de la société dominante (dans certains lieux, les membres entretiennent une relation avec les passants par le biais de la mendicité).

Historique, enfin, « le lieu l'est nécessairement à compter du moment où, conjuguant identité et relation, il se définit par une stabilité minimale. Il l'est pour autant que ceux qui y vivent peuvent y reconnaître des repères qui n'ont pas à être objets de connaissance, [...] ce lieu qu'ont bâti des ancêtres, que les morts récents peuplent de signes [...] » (Augé 1992 : 71). Cet ensemble humain s'est constitué autour d'individus aujourd'hui disparus qui vivaient eux aussi en marge de la société. Et si la mémoire du présent groupe ne remonte pas au-delà de la génération précédente, il reste que la transmission de certaines valeurs de la rue s'explique par la succession d'individus qui ont vécu une même situation, sur un lieu identique. De même que l'exploitant agricole à son fils qui lui succède, le plus ancien du groupe transmet au nouveau venu les valeurs culturelles, les habitudes de vie adoptées inconsciemment, les usages légués par voie orale. Il y a des liens spatiaux, des souvenirs, qui unissent les grands marginaux d'aujourd'hui à ceux d'hier.

\section{Des lieux pour des non-lieux}

Mais si nous parlons de lieux dans ce contexte de la postmodernité (ou de la surmodernité, de laquelle Augé fait l'anthropologie), il faut insister sur la production des non-lieux que produit la société surmoderne. "Si un lieu peut se définir comme identitaire, relationnel et historique, un espace qui ne peut se définir ni comme identitaire, ni comme relationnel, ni comme historique définira un non-lieu » (Augé 1992 : 100). Ainsi, l'auteur soutient l'hypothèse que la surmodernité est productrice de non-lieux, c'est à dire d'espaces qui ne sont pas eux- 
mêmes des lieux anthropologiques et qui n'intègrent pas les lieux anciens :

Un monde où l'on naît en clinique et meurt à l'hôpital, où se multiplient [...] les points de transit et les occupations provisoires (les chaînes d'hôtels et les squats, les club de vacances, les camps de réfugiés, les bidonvilles promis à la casse ou à la pérennité pourrissante), où se développe un réseau serré de moyens de transport qui sont aussi des espaces habités, où l'habitué des grandes surfaces, des distributeurs automatiques et des cartes de crédit renoue avec les gestes du commerce «à la muette », un Monde ainsi promis à l'individualité solitaire, au passage, au provisoire et à l'éphémère (Augé 1992: 101).

Or, ce sont tous ces non-lieux produits par la société surmoderne que les membres du groupe se réapproprient en les inscrivant au sein de valeurs et de références culturelles nouvelles et en s'engageant suffisamment pour leur redonner du sens. La gare, les jardins publics et autres places publiques sont des points de transit et d'occupations provisoires pour les membres de la société dominante. Mais ils sont en même temps, nous l'avons vu, des lieux identitaires, relationnels et historiques pour les membres du groupe. En ce sens, la société surmoderne est toujours productrice de lieux chargés de sens.

\section{Les itinéraires de l'exclusion : parcours quotidiens et parcours de vie}

La référence aux lieux est donc constante. Or c'est la mise en relation de ces différents lieux chargés de sens qui fonde à la fois les itinéraires et l'espace d'exclusion des membres du groupe. Notre propos est à présent de mettre en valeur la construction qui nous a permis de situer sur une carte ces différents itinéraires.

La première étape de cette enquête consistait en la rencontre des principaux organismes et associations caritatives de la ville afin de réaliser des entretiens exploratoires susceptibles d'aider à la réalisation d'une démarche d'insertion dans le milieu d'étude. Ces entretiens exploratoires ont permis d'accumuler quelques renseignements sur le groupe que nous cherchions à suivre, mais aucun intervenant social n'était capable de le situer précisément : "ils traînent quelque part làdedans, mais on ne sait jamais trop où ». Les premières semaines à leurs côtés furent très riches du point de vue de l'enquête. Nous avons en effet très vite remarqué qu'ils suivaient chaque jour le même itinéraire, le même parcours. 


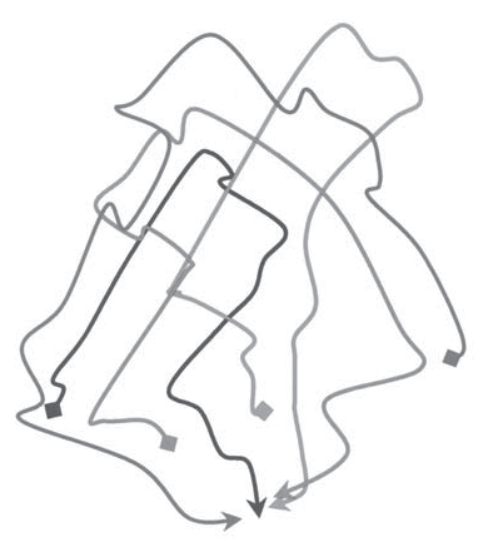

Figure 1 : Entrecroisement des parcours quotidiens au sein de l'espace urbain.

La chaîne des opérations spatialisantes semble donc prendre des références à ce qu'elle produit (une représentation des lieux) ou à ce qu'elle implique (un cadre local). Pour Michel de Certeau, on a ainsi la structure des récits de voyage, mais également, plus largement, de tout autre récit de vie, même si, pour lui, tout récit est un récit de voyage où « des histoires de marche et des gestes sont jalonnés par la citation des "lieux" qui en résultent ou qui les autorisent » (1980: 212). Car il importe en effet de mettre en relation ces itinéraires avec les lieux qui les composent. Dans notre cas, il faut relever deux types de parcours jalonnés par des lieux de référence :

- le parcours quotidien parsemé de places, de jardins, de magasins et de trottoirs chargés de sens pour les acteurs;

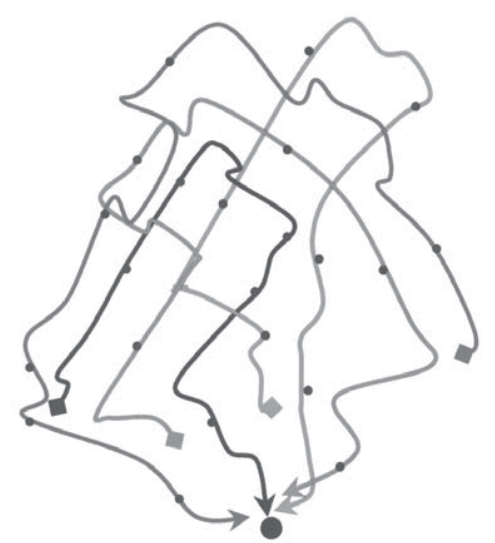

Figure 2 : Des parcours jalonnés de lieux investis quotidiennement. 
— le parcours de vie; la réflexion de Michel de Certeau trouve ici tout son sens. Les récits de vie de ces hommes et de cette femme sont des récits de voyage dont l'écoute renvoie à une référence à certains lieux qui ont marqué l'individu en raison d'une action ou d'un événement dont il a été l'acteur ou le témoin. Ainsi, nous avons souvent entendu Goliath évoquer sa cuisine, lieu auquel il associe la fin de son mariage, puisque c'est au sein de cet espace qu'il a pour la première fois entendu le mot divorce dans la bouche de son ex-femme. Il y a donc une référence constante au lieu qui a participé, de près ou de loin, à la chute dans la rue. Nous l'avons vu, ces quatre sans-abri empruntent chaque jour un itinéraire différent et se retrouvent chaque soir devant la gare. De la même manière, ils ont suivi un "parcours de chute» différent, en fonction de leur place dans la société et des facteurs qui les ont plongés dans ce processus de désaffiliation (Castel 1994), mais se retrouvent tous les quatre en marge de la société qui les a exclus.

\section{Conclusion}

S'il est une question qui a fait l'objet d'un consensus marqué dans le domaine de l'itinérance urbaine, c'est bien celle de la difficulté d'en donner une définition précise (Laberge et Roy 1994). La fragilité du lien social, la précarité de la situation personnelle et la vulnérabilité sur le plan socio-affectif renvoient à une multitude de termes classificatoires et catégorisants. Sans-abri, sans domicile fixe, marginaux, itinérants urbains sont des termes qui soulignent des dimensions particulières: le logement ou son absence, la très grande précarité économique, les difficultés de fonctionnement. Dans cette étude de cas, les constructions sociales marginales de l'espace mettent en relation différents lieux chargés de sens pour l'individu qui s'y engage. L'itinéraire au sein de la ville n'est donc pas aléatoire; il n'est ni subi, ni accidentel. Malgré une alcoolisation excessive, de nombreuses psychopathologies et des traumatismes physiques rarement soignés entraînant une détérioration corporelle foudroyante, les individus développent des stratégies et une organisation spatiales clairement définies et respectées.

Il s'agit donc de rompre avec une vision trop réductrice de cet ensemble humain qui s'organise dans notre rue ou dans notre quartier et qui ne se limite pas à la dimension trop partielle de l'échec social. L'itinérance doit être comprise comme un engagement au sein de certains lieux dont la mise en relation constitue non seulement un itinéraire, mais un espace socialement vécu et ressenti quotidiennement. 


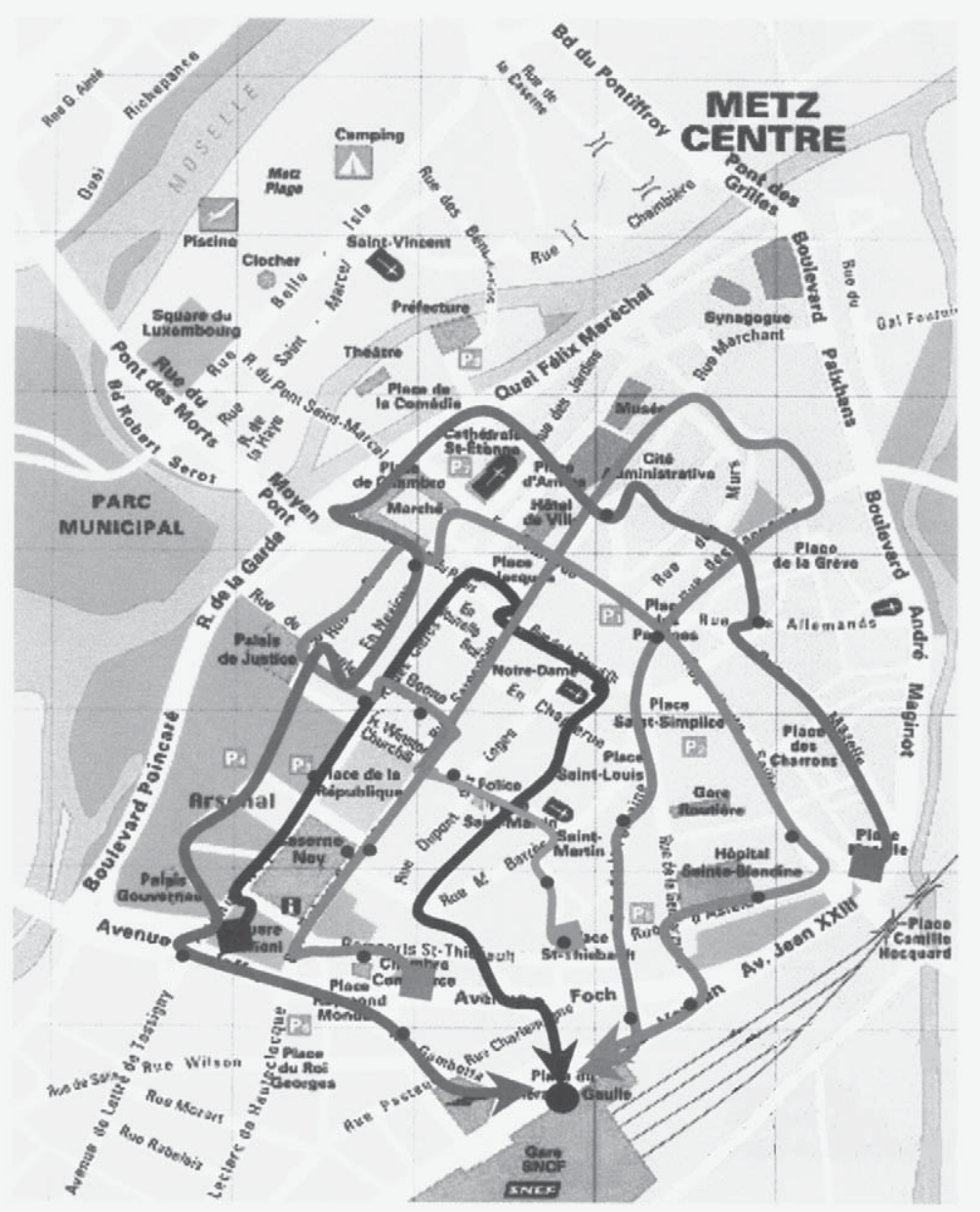

Figure 3 : Les itinéraires de l'exclusion au sein de l'espace urbain marginal.

Le sans-abri, le sans domicile fixe ou le clochard, mal rasé et ivre, dormant dans ses excréments et dans ses vomissures, sous des cartons de récupération devant les grands magasins de la ville, ne saurait alors être vu dans sa dimension figée et instantanée de loque humaine errant sans but au hasard des événements (photographie d'une situation), mais comme un individu socialement exclu qui tente de recréer du sens dans une sous-culture déviante, en venant d'un lieu pour en rejoindre 
un autre (itinéraire). La photographie, prise instantanée, figée et ponctuelle d'une réalité perçue, doit être pensée dans sa dimension mobile et mouvante, en ayant à l'esprit le passé et le futur du moment présentement vécu et perçu par le témoin. 


\section{Références}

Augé, Marc, 1992, Non-lieux. Introduction à une anthropologie de la surmodernité, Paris, Seuil.

Berque, Auguste, 1997, "Logique du lieu et génie du lieu », dans Auguste Berque et P. Nys (dir.), Logique du lieu et oeuvre humaine, Bruxelles, Ousia.

Castel, Robert, 1994, «La dynamique des processus de marginalisation : de la vulnérabilité à la désaffiliation ", Cahiers de recherche sociologique, $22: 11-28$.

Certeau, Michel de, 1980, L'invention du quotidien, Paris, UGE.

Declerck, Patrick, 2001, Les naufragés. Avec les clochards de Paris, Paris, Plon.

Gaboriau, Patrick, 1993, Clochard, l'univers d'un groupe de sans-abri parisien, Julliard, Paris.

Garnier-Muller, Annie, 2000, Les «inutiles»: survivre au quotidien en banlieue et dans la rue, Paris, Éditions de l'Atelier.

Ingold, Tim, 1996, « Hunting and Gathering as Ways of Perceiving the Environment », in R. Ellen et K. Fukui (dir.), Redefining Nature: Ecology, culture and domestication, Oxford, Berg, 117-155.

Laberge Danielle et Shirley Roy, 1994, «Interroger l'itinérance : stratégies et débats de recherche ", Cahiers de Recherche Sociologique, $22: 93-112$.

Meca, Pedro de, 1988, Culture et pauvretés : actes du colloque tenu à la Tourette (L'Arbresle) 13-15 décembre 1985, Paris : Documentation française.

Moreau de Bellaing, Louis et Jacques Guillou, 1995, Les sans domicile fixe : un phénomène d'errance, Paris, L'Harmattan.

Prolongeau, Hubert, 1993, Sans Domicile Fixe, Paris, Hachette.

Radice, Martha Kate, 2000, Feeling comfortable?: les Anglo-Montréalais et leur ville, Sainte-Foy, Presses de l'Université Laval.

Schwartz, Olivier, 1990, Le Monde Privé des Ouvriers. Hommes et Femmes du Nord, Paris, Presses Universitaires de France.

Taesch, René et Denis Robert, 1997, Portraits de groupe avant démolitions, Paris, Stock. 